\title{
Involuntary capture of visual-spatial attention occurs for intersections, both real and "imagined"
}

\author{
BRyan R. BURNHAM AND James H. NeELY \\ State University of New York, Albany, New York
}

\begin{abstract}
In three spatial precuing experiments, we demonstrate attentional capture by an intersection that occurs (1) between two lines that are not part of an enclosed object, and (2) between a line in the cuing array that is not physically present during target search and the invisible circumference of a perceptual circle formed by the elements in the target array. This capture effect conceptually replicates Cole, Gellatly, and Blurton's (2001) corner enhancement effect, in which responses are faster for targets presented near an object's corners rather than along its straight edges. However, it extends that effect by showing that it occurs even when the intersection is not part of an enclosed object and is not physically present during target search. More important, our capture effect occurred even though the target's position was not designated by a perceptually distinctive feature and was not predicted by the intersection's position. Thus, it seems that a line intersection-whether it be real or imaginary - automatically captures visual-spatial attention, contrary to Folk, Remington, and Johnston's (1992) and Gibson and Kelsey's (1998) views that such an involuntary capture of spatial attention is contingent on attentional control settings.
\end{abstract}

An abundance of research has examined whether a visually distinctive and salient event (cue) will involuntarily draw spatial attention to its location, the so-called attentional capture effect (see Yantis, 1993, 2000, for reviews). Evidence for attentional capture is said to occur when responses to a target stimulus are faster and/or more accurate the closer that target's location is to the cue's location. A central issue is whether attentional capture can ever be purely stimulus driven and based on the cue's salience alone (Theeuwes, 1992, 2004; Yantis, 1993, 2000), or whether it is contingent on top-down (goal-directed) influences that direct attention toward a cue only if its distinctive feature is relevant to localizing the target (Bacon \& Egeth, 1994; Folk, Remington, \& Johnston, 1992) or to detecting the target display's appearance (Gibson \& Kelsey, 1998).

In the present research, we seek to demonstrate stimulus-driven attentional capture when the distinctive static feature is the intersection between two lines in an otherwise blank visual field. Importantly, the target's location is neither designated by a distinctive feature nor predicted by the intersection's position in the cuing array. Additionally, the appearance of the target array is not signaled by the appearance of a similar intersection. We chose the intersection of two lines as our cue, because prior research has suggested that line junctures may represent a salient feature that is processed "preattentively." For example, the seminal work of Hubel and Wiesel (1977, 1979) has shown that highly specific hypercomplex cortical cells (in the V2-V4 regions) are activated when inter- sections of a given angle are formed by line segments of specific length. There is also behavioral evidence (Wolfe \& DiMase, 2003) suggesting that cross-like intersections of two lines may be especially salient. That is, a group of plus signs $(+)$ among $L$ s produces rapid texture segregation, and a single + among $L$ s produces a pop-out effect in visual search (see also Julesz \& Bergen, 1983). Although this pop-out effect was shown not to be due to an intersection per se (Wolfe \& DiMase, 2003), but rather to the + (1) being more symmetrical than the $L s$, or (2) having more line terminators than the $L$ s, not knowing the exact reason an intersection represents a salient visual feature does not undermine our using it to elicit attentional capture. Indeed, the findings of Wolfe and DiMase establish that intersections (i.e., multiple line junctures) are salient and can produce a pop-out effect when the + is the target of visual search; however, they do not establish that an intersection between lines will automatically capture attention when the intersection is not the target of search.

Our research is related to Cole, Gellatly, and Blurton's (2001) corner enhancement effect-the finding that the line junctures that form an object's corners receive more attention than its straight edges. For instance, Cole et al. found reaction times (RTs) to a target probe's onset were faster when it appeared near one of the corners of a square "prime" object than when it appeared along one of the prime's straight edges. One possible reason the corner enhancement effect occurs is that an object's intact corners are essential for object identification, because they define the boundaries of geons that make up an object and allow it 
to be identified, as per Biederman's (1987) recognition by components (RBC) theory. Indeed, Biederman found that when corners of simple line drawings were removed, accuracy at reporting those objects' identities was near zero. However, when the corners were intact and equivalent portions of the straight edges connecting the corners were removed, identification accuracy improved dramatically. Hence, corners of objects—or, more simply, line junctures (intersections) - may represent highly distinctive and salient visual features that are assigned attentional priority, because they are crucial for object recognition.

As shown in Figure 1, in the present experiments, observers determined whether a target $R$ or $A$ was present among seven different letters. Prior to the target display, a cuing display was presented. It contained two lines that formed a $90^{\circ}$ intersection at one of eight potential target locations equally spaced on the circumference of an invisible circle. The target appeared where the intersection had occurred (the intersection location-Figure 1A) by chance (i.e., in only one trial of eight); hence, observers had little or no incentive to attend to the intersection. On another random one eighth of the trials, the target appeared where one of the cuing lines had appeared (the line-only location), at the point where it would have crossed the invisible circle opposite to where its intersecting line was tangential to the invisible circle (Figure 1E). This line-only condition is akin to probes appearing along the straight edge of the prime in Cole et al. (2001). On the remaining six of eight trials, the target appeared equally often in one of the six "offline" (uncued) locations. As shown in Figure 1, two each of these six uncued locations were at distances that were either "adjacent" to (panel B), "close" to (panel C), or "far" from (panel D) the intersection's location. If RTs were faster for intersection targets than for line-only and uncued targets, we could interpret this result as a stimulus-driven attentional capture effect that was elicited by the intersection (juncture) of two lines. This is so because the intersection did not predict the target's location; the target was not localized by a distinctive feature, and the target array's appearance was not signaled by an intersection similar to that in the cuing array. Hence, the intersection was irrelevant to localizing the target or signaling the target array's appearance (see Bacon \& Egeth, 1994; Folk et al., 1992; Gibson \& Kelsey, 1998). Additionally, observers were explicitly told to ignore the cuing array altogether.

\section{EXPERIMENT 1}

\section{Method}

Participants. Twenty University at Albany undergraduates reporting normal or corrected-to-normal vision participated for partial research credit for a psychology course.

Design. The design included a single factor with three completely within-participants and randomized levels (cue condition: intersection target, line-only target, and uncued target).

Stimuli. Experiments were programmed and presented using MEL 2.0 (Schneider, 1995) software on a Pentium PC connected to a 15-in. monitor. Participants were tested individually in a small, soundproof, and dimly lit room at a viewing distance of approximately $60 \mathrm{~cm}$ from the monitor.
The background color was black for all of the displays. Cuing displays included two white lines $\left(<0.1^{\circ}\right.$ thick) extending from one edge of the screen to the opposite edge. One line passed through the center of an imaginary circle, which had a radius of $2.35^{\circ}$ and was placed in the center of the display. This line formed a $90^{\circ}$ intersection with the other line, which was the tangent on the invisible circle at one of the eight potential target locations. Target displays included a single white target letter $(R$ or $A)$ and seven white nontarget letters $(S, Z, T, N, U, K$, and $H)$ appearing at equally spaced locations around the circumference of the invisible circle. As described earlier and shown in Figure 1, the target appeared equally often at the intersection, the line-only, the two adjacent, the two close, and the two far locations, giving observers no incentive to attend to the intersection or line-only locations.

Procedure. Participants were told that they would see a display containing a white target letter $(R$ or $A)$ and seven white nontarget letters. They were told to respond as quickly and accurately as possible to the target's identity by pressing either the " $\mathrm{z}$ " or " " key on a standard QWERTY keyboard. (The key assignment for the targets was counterbalanced across participants.) They were also told that the lines presented prior to the target display were irrelevant and should be ignored.

Participants received 16 warm-up trials without the prior appearance of the cuing display, 16 practice trials with the cuing display, and then 384 experimental trials in which 128 possible displayscreated by counterbalancing the two targets by the eight target locations and by the eight intersection locations-appeared three times each in a randomized order. Each trial with a cuing display began with a white fixation dot $\left(0.15^{\circ}\right)$ for $1,000 \mathrm{msec}$, followed by a 100-msec cuing display, a 33-msec blank screen, and a target display that remained visible for 3,000 msec or until a response was made. Participants were given a self-paced break every 64 trials of the session, which lasted approximately $45 \mathrm{~min}$.

\section{Results and Discussion}

A criterion of $15 \%$ errors or less was used as a basis for keeping or excluding a participant's data. No participants made more than 15\% errors in Experiment 1 (the highest was $12 \%$ ). Separate ANOVAs were carried out on RTs and errors. All significant effects are associated with $p<.05$, and are two-tailed.

RTs. Only RTs for correct responses were analyzed. A total of $3.2 \%$ of the trials were excluded because of error. Each participant's geometric mean RT was computed for the intersection-target, line-only target, and uncued-target conditions, both separately for each uncued distance and aggregated across the three uncued-target distances (the aggregated RT was $958 \pm 83 \mathrm{msec}$, with the value following the \pm representing the $95 \%$ confidence interval). Data from the intersection-, adjacent-, close-, far-, and line-only target conditions are presented in the top panel of Figure 2. A one-way ANOVA comparing the mean RTs for the intersection targets, the line-only targets, and the aggregated uncued targets revealed a main effect of cue condition $\left[F(2,38)=15.02, M S_{\mathrm{e}}=2,319\right]$. Planned comparisons indicated that RTs for intersection targets were $50 \pm 39 \mathrm{msec}$ faster than for line-only targets $[t(19)=$ 2.73, SEM = 18.47] and 83 \pm 34 msec faster than for uncued targets $[t(19)=5.09, S E M=16.26]$. RTs were also $32 \pm 20 \mathrm{msec}$ faster for line-only targets than for uncued targets $[t(19)=3.40, S E M=9.50]$, suggesting RTs were facilitated when the target appeared where a line in the cuing display had previously appeared. 


\section{Experiment 1}

Experiment 2

A. Intersection Target

B. Uncued TargetAdjacent
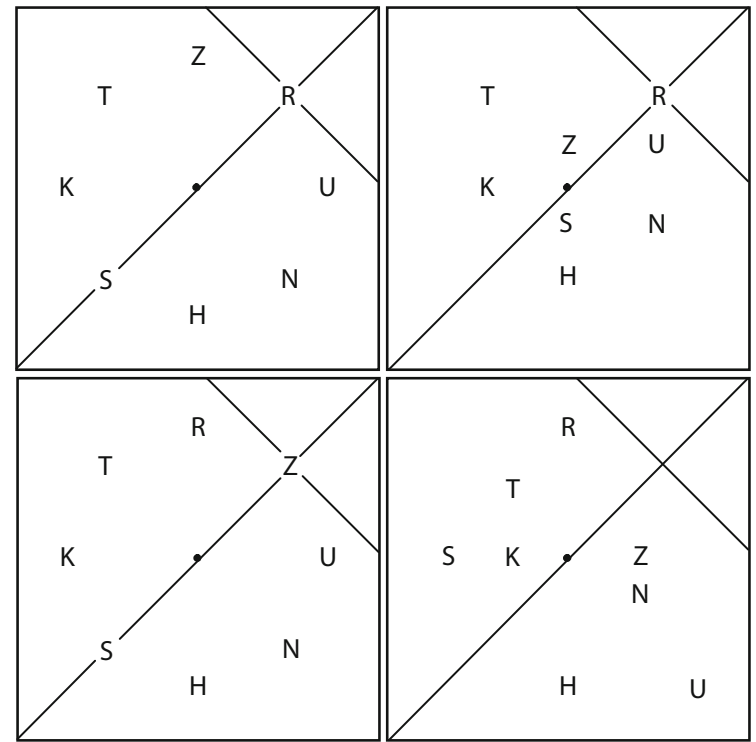

C. Uncued Target-Close

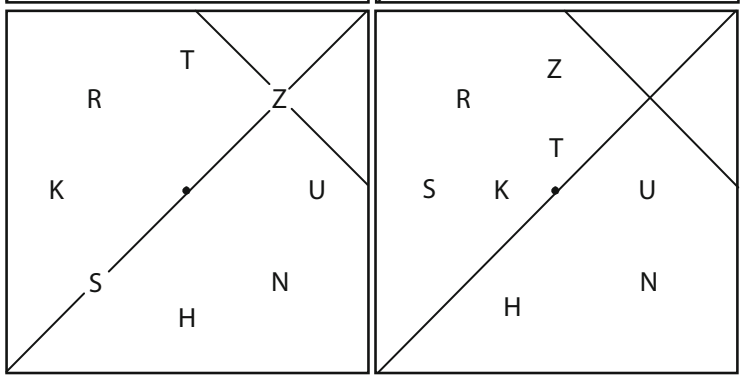

D. Uncued Target-Far

E. Line-only Target
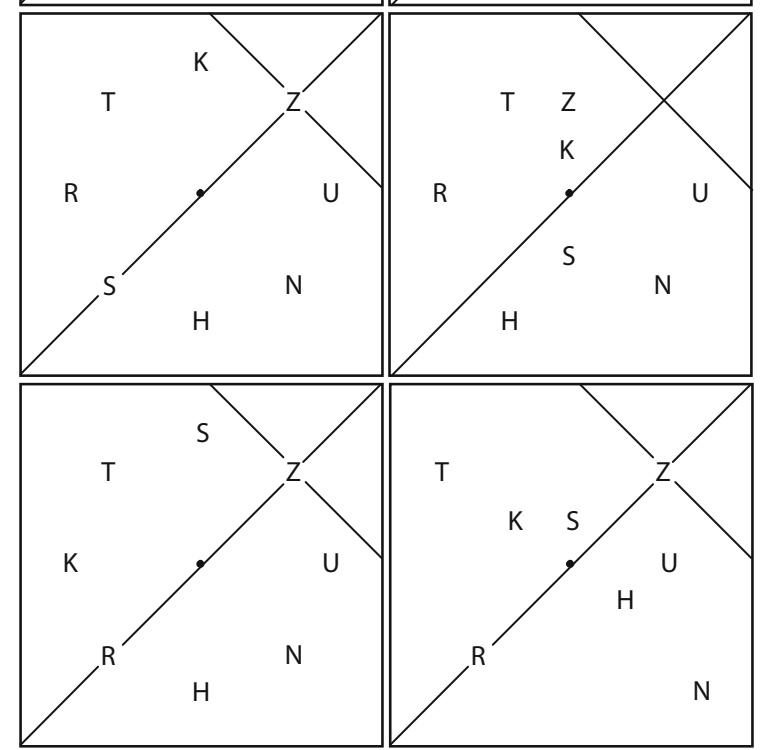

Figure 1. The panels show examples of the target's location in comparison with the intersection appearing at the 1:30 clock position for the five different conditions in Experiments 1 and 2. (The target and the intersection appeared equally often and independently at all eight locations.) The three uncued-target panels on the right were also used in Experiment 3. The intersecting lines preceded the search display.

A one-way ANOVA on the mean RTs for the three uncued target distances (adjacent, close, and far) revealed a distance effect $\left[F(2,38)=27.16, M S_{\mathrm{e}}=693\right]$. As seen in the top panel of Figure 2, RTs increased as the target's distance from the intersection's location increased, suggesting that targets appearing farther from where the 


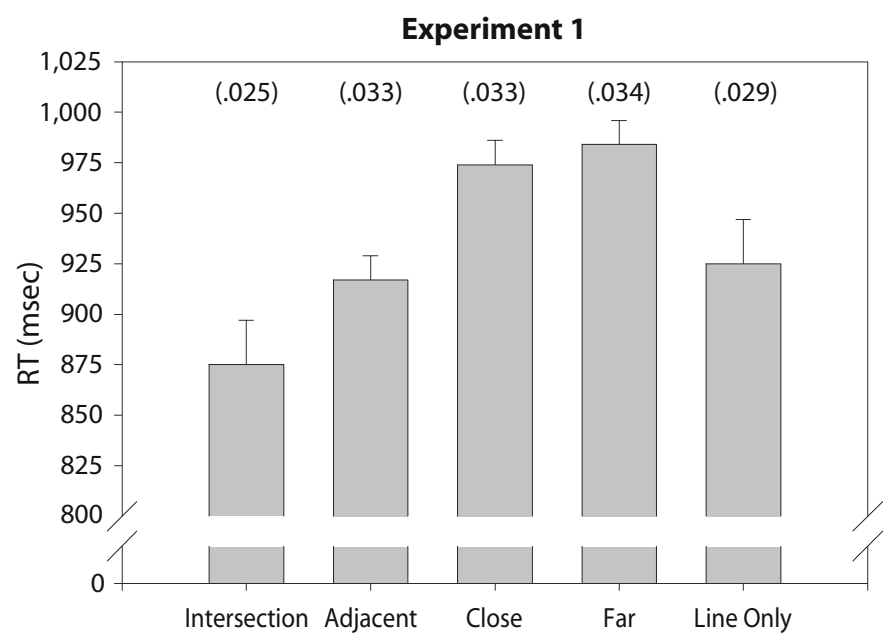

Target Location

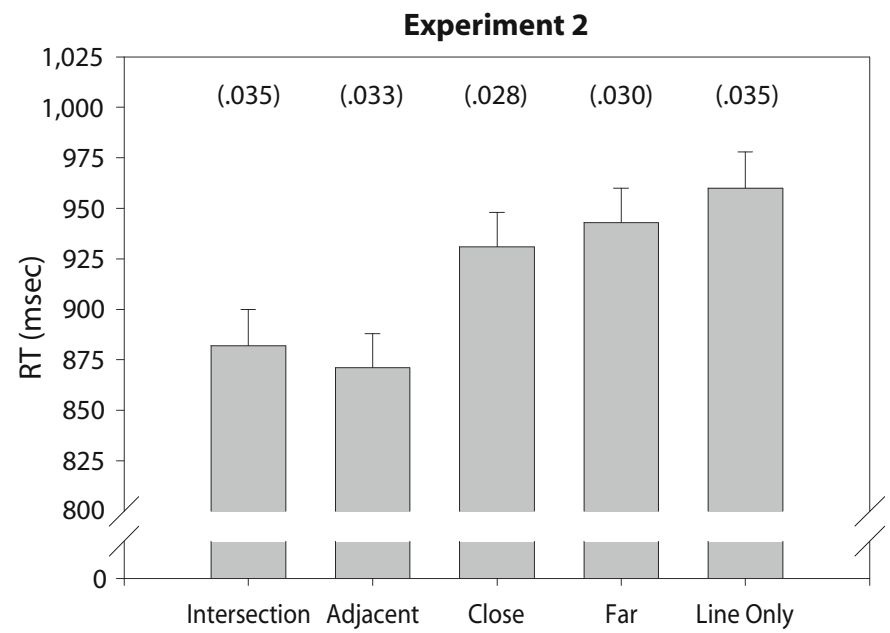

Target Location

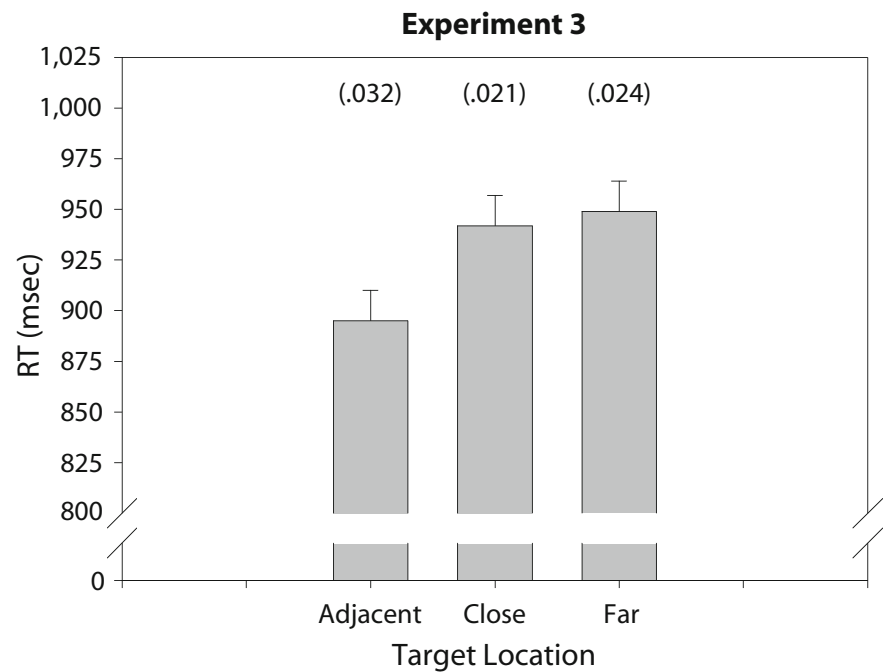

Figure 2. Data from the intersection, adjacent, close, far, and line-only conditions from Experiments 1 and 2 . Only the adjacent, close, and far conditions were tested in Experiment 3. The error bars represent the $95 \%$ confidence intervals based on the within-subjects error term for comparing differences between condition means (Loftus \& Masson, 1994). The values in parentheses are the mean proportions of errors in each condition. 
intersection appeared were more difficult to identifypresumably because they were distant from the focus of the "spotlight" of attention (see Downing \& Pinker, 1985; Shomstein \& Yantis, 2002).

Errors. Error rates were low (all below 3.4\%), with no significant differences among them (all $F \mathrm{~s}<1.5$ ).

Experiment 1 demonstrated that an intersection captured attention, which conceptually replicates and extends Cole et al.'s (2001) corner enhancement effect by showing that it occurs even when that intersection is neither part of an enclosed object nor is physically present during target search. Also, because the intersection did not predict the target's location and was irrelevant to locating the target on the basis of any distinctive feature of the target (Bacon \& Egeth, 1994; Folk et al., 1992), this capture of attention by the intersection appears to be stimulus driven (Theeuwes, 1992; Yantis, 1993, 2000). Perhaps the most surprising result was faster RTs for line-only targets than for uncued targets, even though line-only targets were farthest from the location where the attention-capturing intersection appeared. There are two potential explanations for this. One is that the observers allocated attention to all points along the two lines and not to the intersection per se. By this account, RTs were fastest for intersection targets, not because they appeared at the intersection, but because they received "double attention" because they were near two lines. The much more interesting alternative is that the line-only location was treated as an "imaginary" intersection, where the nontangent line would have intersected with the invisible outline of the circle created by the target display letters. By this account, the faster RTs on line-only targets than on uncued targets represent an "imaginary-intersection" capture effect that is less strong than the "real-intersection" capture effect elicited by the intersection of the two lines in the cuing display. ${ }^{1}$ Experiment 2 tests these two possibilities.

\section{EXPERIMENT 2}

In this experiment, on each trial, the cuing lines appeared exactly as did those in Experiment 1, and two of the letters in the display appeared on the imaginary circle. One was the target and the other was a nontarget on the opposite side of the circle. The remaining six nontargets never appeared on the circumference of the invisible circle, but rather appeared in a randomly chosen subset of locations in a $7 \times 7$ invisible grid. Thus, there was no longer an imaginary circle for the nontangent line to intersect with. If the faster RTs on line-only targets than on uncued targets were due to line-only targets merely being on a line, then RTs for line-only targets should remain faster than RTs for uncued targets, and RTs on intersection targets should remain the fastest, because they still appear near two lines. However, if the faster RTs for lineonly targets than for uncued targets in Experiment 1 were due to line-only targets being presented at an "imaginary" intersection, then RTs on line-only targets should now be slowest, because there was no longer an invisible circle to form an "imaginary" intersection with the cuing, and the line-only target appeared at the location farthest from the real intersection, which should still capture attention.

\section{Method}

Participants. A new sample of 22 undergraduates participated.

Stimuli. Stimuli were the same as those in Experiment 1, with the sole exception that six of the nontarget letters in each display were arranged in a "pseudorandom" pattern within an invisible $7 \times 7$ matrix (Figure 1, right panels). The arrangement of these six letters was different for each trial and is called "pseudorandom" because these six letters never appeared at the fixation location or in any of the eight matrix locations coinciding with the circumference of the invisible circle used in Experiment 1, although distractors could appear on the lines at other locations. The other two letters in each target display were the target letter and the one nontarget letter appearing on opposite sides of the invisible circle that was used in Experiment 1. Otherwise, the stimuli and procedures were identical to those of Experiment 1.

\section{Results and Discussion}

Data from 2 participants were removed due to errors in excess of $20 \%$, with the next highest error rate being $14 \%$. Analyses are based on the remaining 20 observers. Mean RTs were calculated as were those in Experiment 1 and are presented in the middle panel of Figure 2. A total of $3.2 \%$ of the trials were excluded due to error.

RTs. The mean RT for uncued targets aggregated over the three distances was $914 \pm 62 \mathrm{msec}$. A one-way ANOVA revealed a main effect of cue condition $[F(2,38)=19.32$, $\left.M S_{\mathrm{e}}=1,561\right]$. Pairwise comparisons revealed that RTs for intersection targets were $77 \pm 32 \mathrm{msec}$ faster than for line-only targets $[t(19)=5.03, S E M=15.36]$ and $32 \pm 28 \mathrm{msec}$ faster than for uncued targets $[t(19)=2.43$, $S E M=13.20]$, as predicted by both accounts of the Experiment 1 results. However, RTs were now $45 \pm 16 \mathrm{msec}$ slower for line-only targets than for uncued targets $[t(19)=$ 5.92, SEM = 7.63]. This result supports the account that assumes that an "imaginary" intersection captured attention, but to a lesser degree than did the real intersection

A one-way ANOVA on RTs for the three uncued distances revealed a distance effect $\left[F(2,38)=21.59, M S_{\mathrm{e}}=\right.$ $1,360]$, showing that RTs increased as the target's distance from the intersection location increased.

Errors. Errors were low, with no significant differences among them (all $F$ s $<1$ ).

We replicated the intersection attentional capture effect from Experiment 1 and established that the RT benefit for line-only targets in that experiment was not merely due to the target appearing on a line, but rather to its appearing at the location where a line would have intersected with the invisible circumference of the imaginary circle created by the target display stimuli. Moreover, this finding suggests that the fastest RTs on an intersection target were not merely due to the additive effects of the intersection target being near two different lines, but rather were due to the intersection (line junctures) capturing attention. Also, this finding argues against an alternative explanation suggested by a reviewer-that the intersection captured attention not because of its being an intersection per se, but because it was associated with the highest luminance contrast in the display. (This alternative explanation also does not apply 
to Cole et al.'s, 2001, corner enhancement effect study, because in several experiments, the "prime" object was isoluminant with the background and the corner enhancement effect was still obtained.) Specifically, although such a "luminance account" would correctly predict the RT benefit for line-only targets in Experiment 1 (because the edge along the line is associated with a luminance contrast), it also predicts the same benefit for line-only targets in Experiment 2; this was not observed.

\section{EXPERIMENT 3}

In Experiments 1 and 2, observers might have deliberately shifted their attention to the intersection because the target's location and the intersection's locations coincided on some trials. If observers took particular notice of such trials and remembered them better than trials in which the target and intersection did not coincide, then they might have incorrectly assumed that the intersection was predictive of the target's location. If so, then the capture effects in Experiment 2 were not truly involuntary, and thus not purely stimulus driven. In Experiment 3, we addressed this issue by using "random" location displays, as we used in Experiment 2, but never presenting the target in the same location as the intersection. Thus, observers should have no incentive to shift attention to the intersection; if anything, they should shift attention away from it. The target also never appeared in the line-only location, which made it even less likely that observers would use the lines in the cuing display to find the target. If the intersection in the cuing display still (involuntarily) captured attention, RTs for uncued targets should increase as the target-tointersection distance increased.

\section{Method}

Participants. A new sample of 26 undergraduates participated.

Procedure. Experiment 3 was identical to Experiment 2, except that the target never appeared at the intersection or line-only locations.

\section{Results and Discussion}

Data from 4 participants were removed due to error rates in excess of $18 \%$ (with the next highest error rate being $13 \%$ ), and data from 2 participants heard talking across two adjacent rooms during the experiment were also removed. We submitted the data from the remaining 20 participants to separate one-way ANOVAs for the RT and error data for the adjacent, close, and far distances for uncued targets. A total of $2.6 \%$ of all trials were excluded due to error.

RTs. Mean RTs for the adjacent, close, and far distances were $825 \pm 70 \mathrm{msec}, 942 \pm 77 \mathrm{msec}$, and $949 \pm 74 \mathrm{msec}$, respectively, and they are presented in the bottom panel of Figure 2. The distance main effect was significant $\left[F(2,38)=16.52, M S_{\mathrm{e}}=1,028\right]$. Pairwise comparisons indicated that the $46 \pm 23 \mathrm{msec}$ difference between the adjacent and close distances and the $53 \pm 17 \mathrm{msec}$ difference between the adjacent and far distances were significant $[t(19)=4.15, S E=11.18$ and $t(19)=6.50, S E=8.28$, respectively]. The $7 \pm 22 \mathrm{msec}$ difference between close and far distances was not significant $(t<1.0)$, as had also been the case in Experiments 1 and 2. The increasing RTs as a function of the target-to-intersection distance suggest that the intersection still captured attention, even though observers should have been biased against shifting attention toward it, because a target never appeared there.

Errors. Errors for the adjacent, close, and far conditions were $.032, .021$, and .024 , respectively, and the distance main effect was not significant $(F<1)$.

The results are clear: RTs increased as the target's distance from the intersection increased, suggesting that the intersection captured attention, thereby producing faster responses for targets presented in closer spatial proximity to it. This capture effect occurred even though the intersection never coincided with the target's location, suggesting that the shift of attention was truly involuntary and stimulus driven.

\section{GENERAL DISCUSSION}

In three experiments, we found that RTs were faster when a target appeared at the location where an intersection (juncture) of two lines appeared, in comparison with when a target appeared at a point that had been near one of these two lines or at a point away from where both of these lines appeared. However, RTs were not faster when a target appeared at a point near one of these two lines when that line did not intersect with the invisible outline of the circumference of an implied circle that was created by the target display stimuli (line-only condition in Experiment 2). This result suggests that attention was drawn to an intersection (juncture) of two lines rather than to points along individual lines, with more attention being allocated to the location that was near both lines.

There are five important features of our intersection (juncture) attentional capture effect. (1) It occurred when the line junctures forming the attention-capturing intersection were not part of an enclosed "object." This suggests that the attentional capture effect instantiated by Cole et al.'s (2001) corner enhancement effect is not necessarily confined to corners of an enclosed object, but can also occur for simple line intersections (junctures). (2) Our intersection (juncture) capture effect occurred with spatial precuing procedures, such that the intersection was not present with the target. Thus, the results must have been due to the intersection capturing attention rather than to the intersection being used to guide an ongoing visual search. (3) As evidenced by the distance effect in Experiment 3 , the intersection captured attention when the target never appeared at the intersection (or near the straight edge of one line). (4) This capture effect satisfies three criteria for its being considered automatic: (a) The target's location was not designated by a distinctive feature; hence, the intersection was irrelevant to localizing the target; (b) the target never appeared at the intersection's location, but still captured attention in Experiment 3; and (c) the attention-capturing intersection was not present in the target display and, hence, it could not be used to signal the target display's appearance. Thus, we can conclude that the intersection produced an automatic and "noncontin- 
gent" capture of attention. This conclusion runs counter to the view that attentional capture is contingent on features of the capturing event being relevant to locating the target (Bacon \& Egeth, 1994; Folk et al., 1992) or to detecting the appearance of the target display (Gibson \& Kelsey, 1998). ${ }^{2}$ To our knowledge, our capture effect is the first to satisfy all three of the foregoing criteria for automaticity when the stimulus capturing attention is a static discontinuity. This automatic capture of attention is likely due to line intersections (junctures) being critical for determining object identity (Biederman, 1987), thereby causing them to be assigned high attentional priority even when they are not part of an enclosed object. This is similar to attentional priority being assigned to the abrupt onset of a new item (Yantis \& Jonides, 1984). (5) The final-and perhaps most interesting - aspect of our data is that attentional capture occurred for an "imaginary" intersection between an invisible line and the "invisible" implied circumference of a circular percept. This capture effect also satisfies the criteria for its being an involuntary, stimulusdriven capture of attention. Thus, it seems that visualspatial attention is automatically attracted to intersections, both real and imagined.

\section{AUTHOR NOTE}

We thank Geoff Cole, James Cutting, Patrick O'Connor, Jay Pratt, Chris Olivers, and an anonymous reviewer for helpful comments on an earlier version of this article. Correspondence regarding this article should be addressed to B. R. Burnham, who is now at the Department of Psychology, Alumni Memorial Hall, University of Scranton, Scranton, PA 18510 (e-mail: attention.perform@gmail.com).

\section{REFERENCES}

BACON, W. F., \& EgETh, H. E. (1994). Overriding stimulus-driven attentional capture. Perception \& Psychophysics, 55, 485-496.

Biederman, I. (1987). Recognition-by-components: A theory of human image understanding. Psychological Review, 94, 115-147.

Cole, G., Gellatly, A., \& Blurton, A. (2001). Effect of object onset on the distribution of visual attention. Journal of Experimental Psychology: Human Perception \& Performance, 27, 1356-1368.

Downing, C., \& PINKER, S. (1985). The spatial structure of visual attention. In M. I. Posner \& O. S. M. Marin (Eds.), Attention and performance XI (pp. 171-188). Hillsdale, NJ: Erlbaum.

Folk, C. L., Remington, R. W., \& Johnston, J. C. (1992). Involuntary covert orienting is contingent on attentional control settings. Journal of Experimental Psychology: Human Perception \& Performance, 18, 1030-1044.

GiBSON, B. S., \& Kelsey, E. M. (1998). Stimulus-driven attentional capture is contingent on attentional set for displaywide visual features. Journal of Experimental Psychology: Human Perception \& Performance, 24, 699-706.

Hübel, D. H., \& Wiesel, T. N. (1977). Functional architecture of macaque monkey visual cortex. Proceedings of the Royal Society of London, 198, 1-59.
Hubel, D. H., \& Wiesel, T. N. (1979). Brain mechanisms of vision. Scientific American, 241, 150-162.

JuLESZ, B., \& BERGEN, J. R. (1983). Textons, the fundamental elements in preattentive vision and perception of textures. Bell System Technical Journal, 62, 1619-1645.

Loftus, G. R., \& Masson, M. E. J. (1994). Using confidence intervals in within-subject designs. Psychonomic Bulletin \& Review, 1, 476-490.

ScHNEIDER, W. (1995). Micro Experimental Laboratory (Version 2.0) [Computer software]. Pittsburgh, PA: Psychology Software Tools.

Shomstein, S., \& Yantis, S. (2002). Object-based attention: Sensory modulation or priority setting? Perception \& Psychophysics, 64, 41-51.

THEEUWES, J. (1992). Perceptual selectivity for color and form. Perception \& Psychophysics, 51, 599-606.

THEEUWES, J. (2004). Top-down strategies cannot override attentional capture. Psychonomic Bulletin \& Review, 11, 65-70.

Wolfe, J. M., \& DiMase, J. S. (2003). Do intersections serve as basic features in visual search? Perception, 32, 645-656.

YANTIS, S. (1993). Stimulus-driven attentional capture and attentional control settings. Journal of Experimental Psychology: Human Perception \& Performance, 19, 676-681.

YANTIS, S. (2000). Goal-directed and stimulus-driven determinants of attentional capture (tutorial). In S. Monsell and J. Driver (Eds), Control of cognitive processes: Attention and performance XVIII (pp. 73103). Cambridge, MA: MIT Press.

YAntis, S., \& Jonides, J. (1984). Abrupt visual onsets and selective attention: Evidence from visual search. Journal of Experimental Psychology: Human Perception \& Performance, 10, 610-621.

\section{NOTES}

1. We thank Tram Neill for suggesting this capture of attention by an "imaginary intersection."

2. A die-hard proponent of Gibson and Kelsey's (1998) view could argue that our capture was contingent on an observer's attentional set for "line junctures," "lines," and "line terminators," because these features made up the letters in the target array and, hence, could be used to signal the appearance of the target array. If an attentional set for these features was adopted, the line intersection (juncture) in the cuing array may have captured attention because the intersection was relevant to signaling the target array's appearance. This account seems implausible, because the line junctures in the letters and the line juncture in the cuing array were highly dissimilar. Moreover, because this is such a stretch of their hypothesis, we believe that Gibson and Kelsey's account is rendered virtually unfalsifiable by accepting this line of reasoning to argue that our capture effect was contingent on the observer's attentional set. Another related possibility brought up by a reviewer is that our capture effects were not stimulus driven, because line intersections are in the observer's attentional set because the target letters $(A$ and $R$ ) contain line intersections. However, because the nontarget letters also have line intersections, our capture effects do indeed run counter to the original formulation of Folk et al.'s (1992) contingent orienting hypothesis, which states that in order for a stimulus to capture attention, the feature leading to the capture must be relevant to localizing the target.

(Manuscript received April 27, 2006; revision accepted for publication August 24, 2006.) 